\title{
The Research of Vibration Suppression Method for Silicon Micro-machined Gyro Driven by the Rotation Aircraft
}

\author{
Zengping Zhang ${ }^{1, a}$, Fuxue Zhang ${ }^{2, b}$, Wei Zhang ${ }^{2, c}$, Yinjuan Gao ${ }^{2, d}$ \\ ${ }^{1}$ School of electronic Engineering, Beijing University of Posts and Telecommunications, Beijing \\ 100876, China \\ ${ }^{2}$ Sensing Technique Research Center, Beijing Information Science and Technology University, \\ Beijing 100101, China

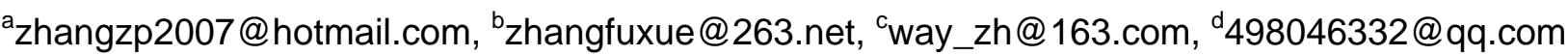

Keywords: silicon micro-machined gyro; silicon pendulum; rudder surface; angular velocity.

\begin{abstract}
With the practical application of silicon micro-machined gyro driven by rotation aircraft, it is found that the rotation aircraft's rudder surface vibration significantly influences the performance of the gyro. In this paper, as the background of the silicon micro-machined gyro, the vibration mechanism is analyzed according to the mathematics model and dynamics. Under the influence of vibration, both of the silicon pendulum's stress form and output signal are obtained. The paper proposes a reasonable installation method to suppress the vibration. The experimental result shows that the method is effective to reduce the gyro's output signal noise. And the result is good.
\end{abstract}

\section{Introduction}

Silicon micro-machined gyro driven by rotation aircraft is driven itself by using of rotating of rotation aircraft. The Pitch angular velocity or yaw angular velocity of the aircraft, which is perpendicular to spin angular velocity of the aircraft, generated the Coriolis force. It is sensitive to aircraft's pitch or yaw angular velocity. Without driving part for this gyro, the structure is simple and it is easy to process. Therefore, it is very suitable for mounting on the rotation aircraft and become a sensitive component of the rotation aircraft control system. From the practical application perspective, the rotation aircraft's rudder surface deflection produces vibration force, which makes its silicon pendulum stress change. The vibration force makes the output signal noise increase significantly and the performance of the gyro output signal becomes worse.

\section{Silicon micro-machined gyro working principle}

Silicon pendulum structure principle of silicon micro-machined gyro is shown as Fig.1. The $a x_{w} z_{q}$ is the yaw or pitch coordinate system. The origin of the coordinate system is chosen in center of silicon mass. $o z_{0}$ is the axis of rotation of the aircraft. The direction is pointing to the front of the aircraft. $a x_{0}$ is in the vertical symmetry plane of the aircraft, and vertical with $a z_{0}$. The direction is upward. $\sigma x_{0}$ is vertical with the plane $u x_{0} \psi_{0}$. The direction is determined by the right hand coordinate system. Coordinate system $a x_{0} \beta_{0} z_{0}$ is no rotation when the aircraft spins.
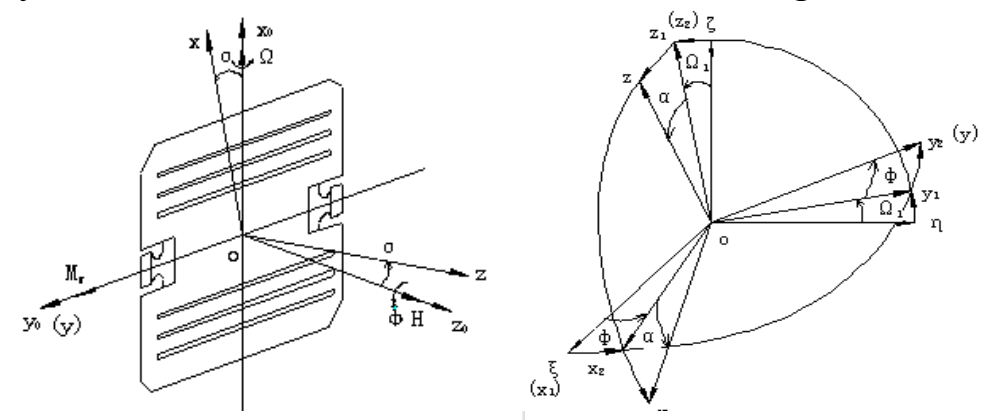

Fig.1 The structure principle diagram

Fig.2 Coordinate Transformation 
Coordinate system $a x y z$ is fixed with the silicon pendulum. The origin is in the center of mass. Initially oz coincides with axis of the aircraft spin. The direction points to the front of the aircraft. It is known as the drive shaft. $o x$ is in the vertical symmetry plane of the aircraft, and vertical with oz. The direction is upward. It is called the input shaft, which is also the aircraft's yaw or pitch axis. $\otimes y$ places through the silicon torsion beam axis of symmetry, and its direction determined by the right hand coordinate system, called the output shaft. With the rotation of the silicon pendulum, coordinate system $v x y z$ is rotating together. The $\dot{\psi}$ is the angular velocity of the rotation aircraft. The $\boldsymbol{\Omega}$ is the pitch or yaw angular velocity of the aircraft. The $\boldsymbol{\alpha}$ is the angular velocity of silicon pendulum vibration around the output shaft.

The working principle is based on the output shaft torque balance. When the aircraft spins, silicon pendulum rotates around the drive shaft with $\phi$ (spin angular velocity). Meanwhile, yaw or pitch angular velocity $\Omega$ is inputted in the input shaft. The Coriolis force is generated causing gyro torque $\boldsymbol{M}_{g}$ in output shaft.

$$
M_{g}=H \times \Omega
$$

Under the action of the gyro torque, the silicon pendulum will turn around the output shaft, for $\alpha$ corner. It also leads to the damping torque $M_{d}$ and the elastic support beam torque $M_{s}$ along the $a y$ negative direction. Their mathematical expressions are given below

$$
\begin{gathered}
M_{d}=D \dot{\alpha} \\
M_{s}=K_{\tau} \alpha
\end{gathered}
$$

$D$ is the damping coefficient, $K_{T}$ is the elastic support beam torsional stiffness. Total torsional rigidity can be obtained by elastic mechanics.

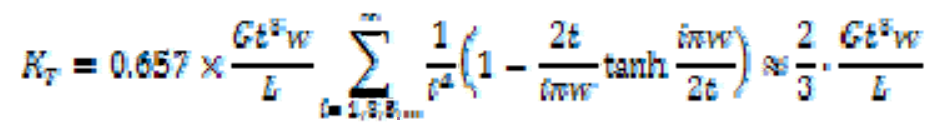

The rectangular plane with length $A$ and width $B$ is relevant to the bottom surface movement, and the gap between them is $h$. The damping coefficient is shown.

$$
D=\frac{F_{\text {davgp }}}{d^{2} h / d^{2} t}=\frac{A B^{2} g E}{h^{2}}\left[1-\frac{192 B}{A \pi^{2}} \sum_{n=1,7 B}^{\infty} \frac{1}{n^{2}} \tanh \frac{n \pi A}{2 B}\right]
$$

$\mu$ is the gas viscous coefficient. The series can be fast convergence, using only the first

$$
D=\frac{F_{\text {damp }}}{d h / d t} \approx \frac{A B^{*} d x}{h^{2}}\left[1-\frac{192 B}{A \pi^{2}} \tanh \frac{n \pi A}{2 B}\right]
$$

Thus equation is

$$
l *=M_{g}-M_{d}-M_{s}
$$

Eq. (1) to Eq. (3) substitute into Eq. (7), we can obtain

$$
l \alpha+D \alpha+K_{\tau} \alpha=H \times \Omega
$$

$\boldsymbol{I}$ is moment of inertia which silicon pendulum around the output shaft. $\boldsymbol{H}$ is angular momentum of silicon pendulum. So the swing of a silicon pendulum is a two order linear differential equations.

Coordinate transformation can describe the Eq. (8). As shown in Fig.2, $05 \eta \%$ is the inertial frame, $\propto x_{1} y_{1} z_{1}$ is yaw or pitch coordinate system, $\alpha x_{2} y_{2} z_{2}$ is aircraft spin coordinate system, $\propto x y z$ is the coordinate system fixed with silicon pendulum. 
According to the theorem of moment of momentum, in the inertial coordinate system $\alpha \xi q \xi$, the equation can be obtained based on the rigid body of fixed-point rotation.

$$
\frac{\mathrm{d}}{\mathrm{dt}}\left[\begin{array}{l}
G_{\xi} \\
G_{q_{q}} \\
G_{\xi}
\end{array}\right]=\left[\begin{array}{l}
M_{k} \\
W_{q} \\
W_{k}
\end{array}\right]
$$

Silicon pendulum moment of momentum is $\left[\begin{array}{l}G_{\mathrm{k}} \\ G_{\mathrm{k}} \\ G_{\mathrm{i}}\end{array}\right]=\zeta\left[\begin{array}{l}\omega_{\xi} \\ \omega_{k} \\ G_{\xi}\end{array}\right]$.

The suffered moment of the silicon pendulum is $\left[\begin{array}{l}M_{\xi} \\ M_{\eta} \\ M_{C}\end{array}\right]$.

7 is the silicon pendulum moment of inertia, which is a constant matrix.

In the inertial coordinate system $\operatorname{ain} \eta$, around $a \xi$ with angular velocity $\boldsymbol{\Omega}$, the silicon pendulum rotates $\Omega t$ angle to yaw or pitch coordinate system $\alpha x_{1} z_{1} z_{1}$. In $\alpha x_{1} y_{1} z_{1}$, with the angular velocity $\phi$ around $c z_{1}$, it rotates to $2 x_{2} y_{2} z_{2}$. The angle is $p^{2}$. Then in $a x_{2} y_{2} z_{2}$, around $o y_{2}$ with angular velocity $d$, it rotates $a$ angle to axyz coordinate system. Through three cosine matrix transformation, the specific dynamic equation is shown as below

$$
J_{y} d+D d x+\left[\left(J_{z}-J_{x}\right) \phi^{2}+K_{\gamma}\right] \alpha=\left(J_{z}+J_{y}-J_{x}\right) \Omega \phi \cos (\phi t)
$$

$I_{x}, I_{y}, I_{z}$ are inertia moments which are respectively in the $o x, o y, o z . E_{T}$ is the silicon pendulum elastic support beam torsional stiffness, and $D$ is the damping coefficient. To simplify the Eq. (10)

In formula

$$
d+2 \xi \omega_{0} d t+\omega_{0}^{2} \alpha=f_{0} \cos (\phi t)
$$

$$
\begin{aligned}
& \omega_{y}^{2}=\frac{1}{J_{y}}\left[\left(V_{z}-J_{x}\right) \phi^{2}+K_{\tau}\right]
\end{aligned}
$$

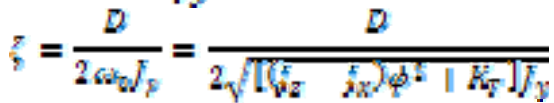

$$
\begin{aligned}
& f_{0}=\frac{1}{J_{y}}\left(J_{z}+J_{y}-J_{x}\right) A_{\phi}
\end{aligned}
$$

The solution of equation consists of two parts. One is free vibration, the other is forced vibration. The free vibration is the transient solution of the equation, which is completely determined by the initial conditions. The calculation is

$$
\alpha=A e^{-n t} \cos \left(\sqrt{\omega_{0}^{2}-n^{2}} t+0\right)
$$

A is the integral constant, $\mathrm{n}$ is the attenuation factor $\left(n=\xi \omega_{0}\right)$.

The steady state solution of the equation is as shown

$$
\alpha=\frac{\left(V_{z}+J_{y}-J_{x}\right) \mu_{\phi}}{\sqrt{\left.\left[\left(J_{z}-J_{x}-J_{y}\right) \phi^{2}+K_{z}\right]^{2}+(D)^{2}\right)^{2}}} \cos (\phi t-\beta)
$$

$\beta=\tan ^{-1} \frac{2 n \phi}{\omega_{0}{ }^{2}-\phi^{2}}$ is a phase angle which is silicon pendulum vibration behind the driving force.

\section{Rotation aircraft rudder surface working principle}




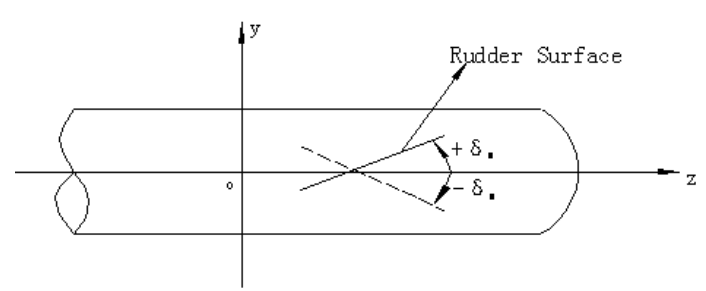

Fig. 3

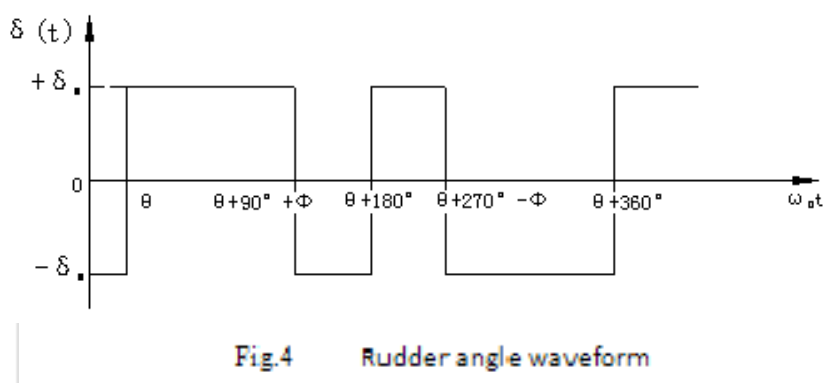

The rotation aircraft rotates around its longitudinal axis, using a steering engine and a pair of rudders. Through the control of rudder commutation time, the control force in any direction of the space is produced to manipulate aircraft pitch and yaw direction movement. The rudder and rudder angle is shown in Flg $3^{[2]}$.

The steering gear is a relay actuator. It controls rudder deflection. The rudder's control signal is equal amplitude width modulation pulse signal. The mathematical model of this square wave signal is shown in Eq. (14).

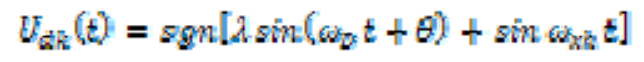

$\omega_{D}$ is the angular frequency of the aircraft spin, $\theta$ is the initial phase, $\omega_{x \mathrm{~h}}$ is the angular frequency of the linear signal, and $\lambda=\frac{A_{l i p}}{A_{x \in \hbar}}$ is the ratio of control signal's amplitude and linear signal's amplitude.

In practice, the angle frequency of the linear oscillator signal is often 2 times the frequency of the aircraft spin and more. The stability of the spin frequency is $f_{5}$ when the aircraft is flying, the angle frequency of the linear oscillator signal is $2 f_{p}$ and more.

If $U_{d \tilde{d}_{i}}>0$, rudder angle is $+\delta_{i n}$, conversely, if $U_{d \hbar}<0$, the rudder angle is $-\delta_{\mathrm{min}}$. When rudder control signal changes its sign, the rudder immediately changes from a limit position to another limit position. Equation as follows

$$
\varepsilon(t)=\varepsilon_{v i} \operatorname{sgn}\left[\lambda \sin \left(\omega_{0} t+\theta\right)+\sin \left(2 \omega_{v i} t\right)\right]
$$

From the above equation, it is known that the aircraft rotates a cycle, rudder reverses four times. $\theta$ is the initial phase of the rudder's control signal. $\theta$ is decided by the target azimuth. $\Phi$ is the phase Angle of the rudder change. $\delta_{m}$ is the limit of rudder angle.

Therefore, the introduction of linear oscillation signal which two times the spin frequency, then limiting, shaping, amplification, the $\bar{\partial}(t)$ is as shown in F:g. $4^{2}$.

\section{Analysis of rudder partial force}

The so-called the rudder partial force refers to the aircraft rudder surface impact on the aircraft in the instantaneous moment. Because rudder surface is radians, interaction is point touching. So rudder partial force can be equivalent to the impulse signal. The direction of force is determined by rudder surface deflection. Polar of $\hat{\delta}_{\mathrm{m}}$ can change the direction of force. The rudder partial force's frequency is $f_{r}$. With the rudder surface deflection, the force is as shown in Fig.5.

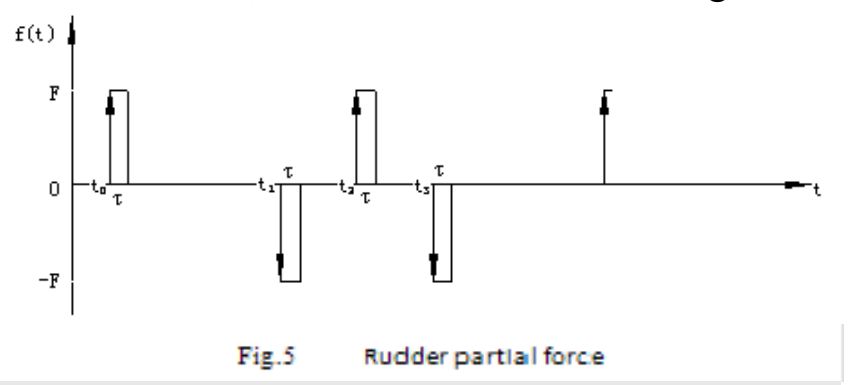


Rudder partial force is a periodic signal. Using the method of harmonic analysis, the force can be expanded into a series of different frequency harmonic vibration, which is shown in Eq. (16).

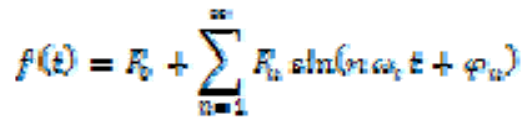

$F_{0}, \quad F_{n}, \varphi_{\mathrm{r}}$ are constants, $\omega_{\mathrm{r}}=2 \pi f_{\mathrm{r}}(\mathrm{rad} / \mathrm{s})$.

In order to analyze conveniently, we should take the first harmonic and the second harmonic such as Eq. (17) and Eq. (18). The first harmonic frequency is $f_{r}$. The second harmonic frequency is $2 f_{r}$.

$$
\begin{aligned}
& F_{1}(t)=F_{1} \sin \left(\omega_{2} t+\varphi_{1}\right) \\
& F_{2}(t)=F_{2} \sin \left(2 \omega_{2} t+\varphi_{2}\right)
\end{aligned}
$$

\section{A. The influence of the first harmonic}

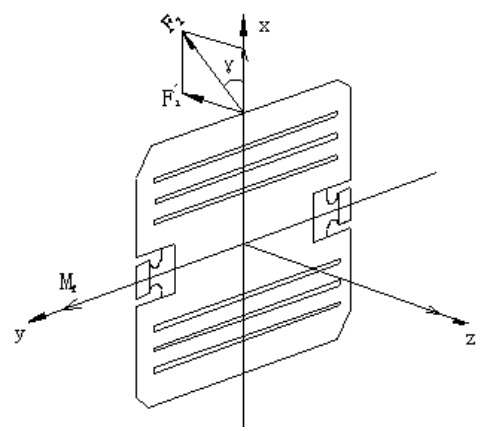

Fig.6 stress vector decomposition in the sillicon pendulum

Fig. 6 is the stress condition of the silicon pendulum. As the restrictions of the production process of silicon micro-machined silicon pendulum, the silicon pendulum always have a certain angle with the horizontal planes, which will produce force vector decomposition.

$$
F_{1}^{\prime \prime}(t)=F_{1}^{\prime} \sin \left(\omega, t+\varphi_{1}\right)
$$

$F_{1}=F_{1} \sin \gamma, \quad \gamma$ is a angle between silicon pendulum and the horizontal plane.

This force makes silicon pendulum doing the simple harmonic motion. From Eq. (11) we can obtain a two order linear differential equation.

$$
\alpha+2 \tau \omega_{0} d+\omega_{0}^{2} \alpha=F_{1}{ }^{\prime} \operatorname{sln}\left(\omega_{r} t+\varphi_{1}\right)
$$

From Eq. (20), we can solve a steady-state solution such as Eq. (21).

$$
\alpha(t)=B \sin \left(\omega_{r} t+\varphi\right)
$$

Eq. (21) is the silicon pendulum vibration that is caused by the fundamental frequency. The vibration will be added to standard signal. This will lead to the gyro output signal distortion. Fig.7 is the simulation results of the above analysis and Fig. 8 is the experimental results. It can be seen from the two figures that the experimental results and simulation results are basically the same. 

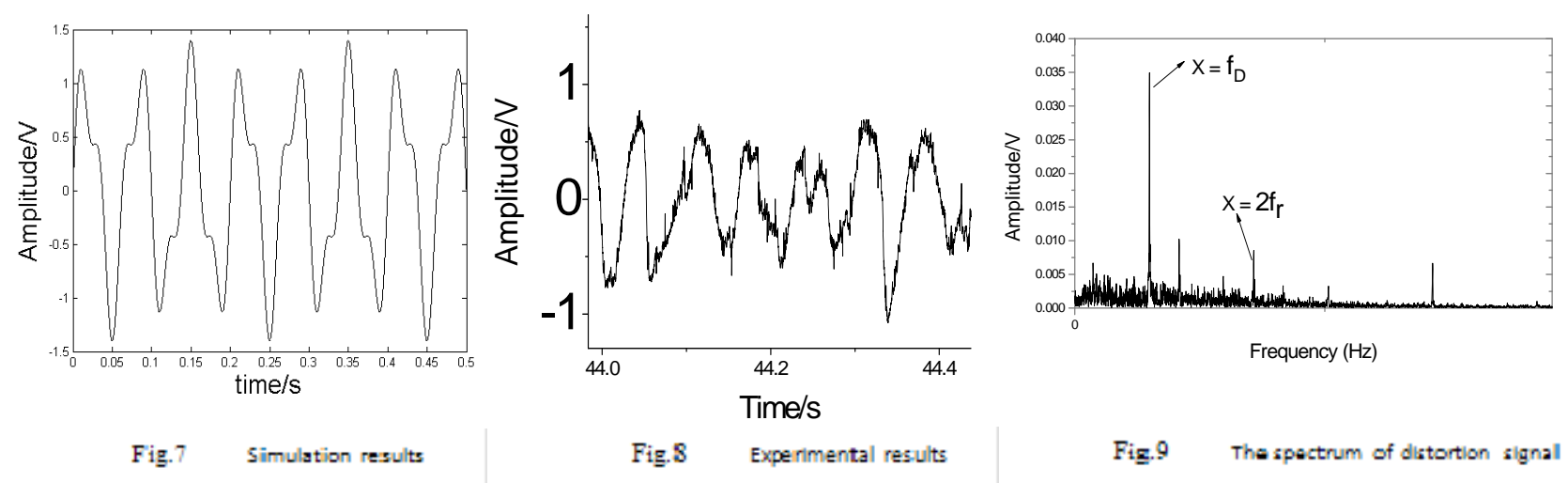

Fig.9 is a spectrum diagram obtained from the experimental results. There is a distinct spectrum at the point of $f$. And it has been verified that the fundamental vibration will cause the gyro output signal distortion under the installation method of Fig.6.

\section{B. The influence of the second harmonic}

It is known that the silicon micro-machined gyro is a two order linear system. We do Laplace transform for the equation to obtain system transfer function.

$$
F(g)=\frac{\omega_{0}^{2}}{g^{2}+2 \omega_{0} g+\omega_{0}^{2}}
$$

$\omega_{0}$ is the natural frequency, $\zeta$ is the damping ratio. According to Eq. (22), we can calculate system amplitude frequency and phase frequency characteristics.

$$
\begin{aligned}
& A(\omega)=\frac{1}{\sqrt{\left[1-\left(\frac{\omega}{\omega_{0}}\right)^{2}\right]^{2}+\left(2 \xi \frac{\omega}{\omega_{0}}\right)^{2}}} \\
& \phi(\omega)=-\tan ^{-1}\left[\frac{2 \zeta \frac{\omega}{\omega_{0}}}{1-\left(\frac{\omega}{\omega_{0}}\right)^{2}}\right]
\end{aligned}
$$

From Eq. (23) to Eq. (24), it can be seen that the frequency characteristics depends on silicon micro-machined gyro's natural frequency $\omega_{0}$ and the damping ratio $\zeta$. If $\xi \ll 1, \omega_{0} \delta \omega$, then $A(\omega) \approx 1$ and $\phi(\omega)$ is very small. Table 1 shows the experimental data. Fig.10 is the

\begin{tabular}{|c|c|c|c|c|c|c|c|c|c|c|c|c|c|}
\hline$\vec{F}(\overline{A i})$ & \rfloor & 9.95 & $M, D$ & $N W$ & X. & M & 1719 & $4.7]^{\prime}$ & 5.6 & 597 & 6n $\lambda$ & 79.67 & 4.5 \\
\hline $\mathbb{Z}_{-}(m)$ & 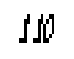 & $\eta$ & 6 & 5 & 5 & $d a$ & 4 & $\|$ & $\$ 5$ & $d 7$ & J & 59 & 5 \\
\hline
\end{tabular}
amplitude-frequency characteristic curve measured experimentally.

Table 1 Amplitude-frequency characteristics experimental data 


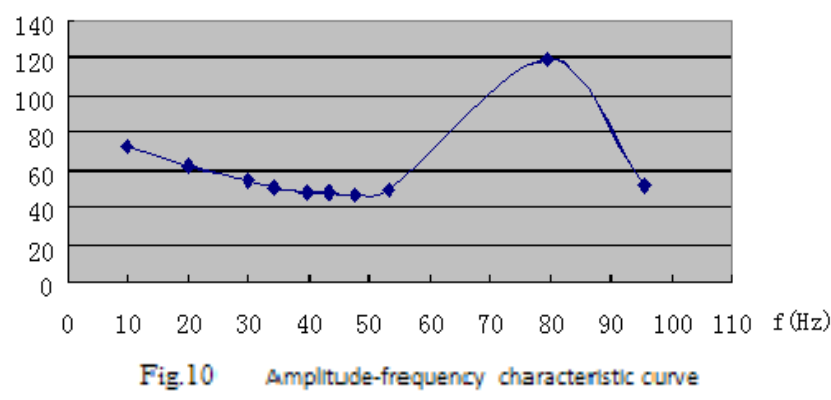

From the experiment data, it is known that the silicon micro-machined gyro natural frequency is close to $68.24 \mathrm{~Hz}$. The second harmonic frequency is $2 f_{r}$. It is very close to the natural frequency which means that the second harmonic is likely to cause resonance. Fig.11 is the time-domain waveform tested by experiment. Fig. 12 is the frequency spectrum diagram corresponding to the Fig. 11, in which a large amplitude spectrum line appears at $2 f_{p}$. This is generated by resonance and makes the time-domain waveform appearing irregular beating such as Fig.11.
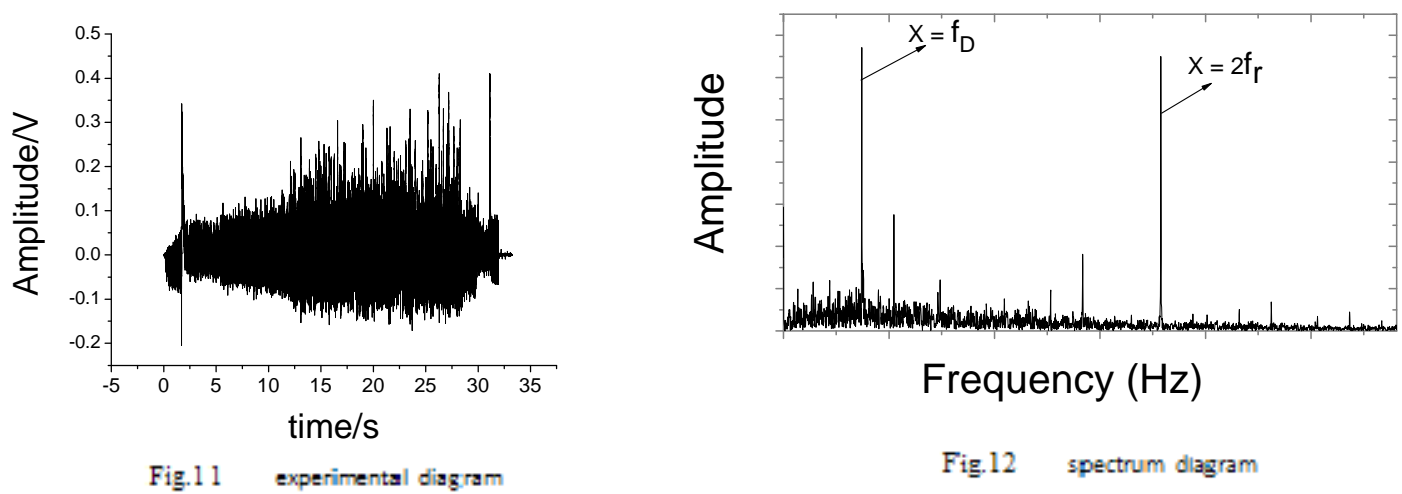

\section{The method to suppression influence of rudder partial force}

The method of suppression.Fig.13 is a stress schematic figure that is the silicon pendulum to rotate 90 degrees when installed. From the figure it is known that the stress direction is parallel to the output shaft of the silicon pendulum. So it can't form torsional moment. And the method effectively eliminates the superposition interference of $\frac{i}{i r}$ and resonance caused by $2 f_{r}$.

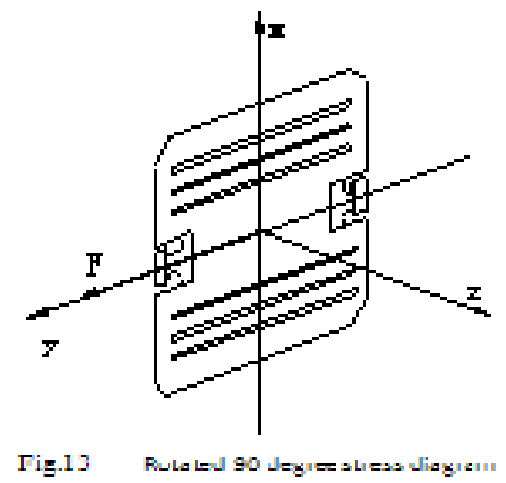

Experimental validation and analysis.According to the results of the analysis in this paper, we make a test in a kind of high precision experimental platform. Fist, we give a $f_{b}$ rotation as driver to make silicon pendulum having an angular momentum, and join a transverse angular velocity of $90^{\circ} \mathrm{fs}$. Second, putting a liner signal of $f$ to make the rudder surface deflection, which causes the rudder partial force. Third, changing the transverse angular velocity range from $90^{\circ} / \mathrm{k}$ to $180^{\circ} / \mathrm{s}$. Last, stopping the transverse angular velocity and backing to zero. In all phases rudder partial force is the same. At the same time the output signal is sampled with $10 \mathrm{kHz}$ sampling frequency to obtain the output waveform. The experimental results are shown in Fig.14 to Fig .17. 


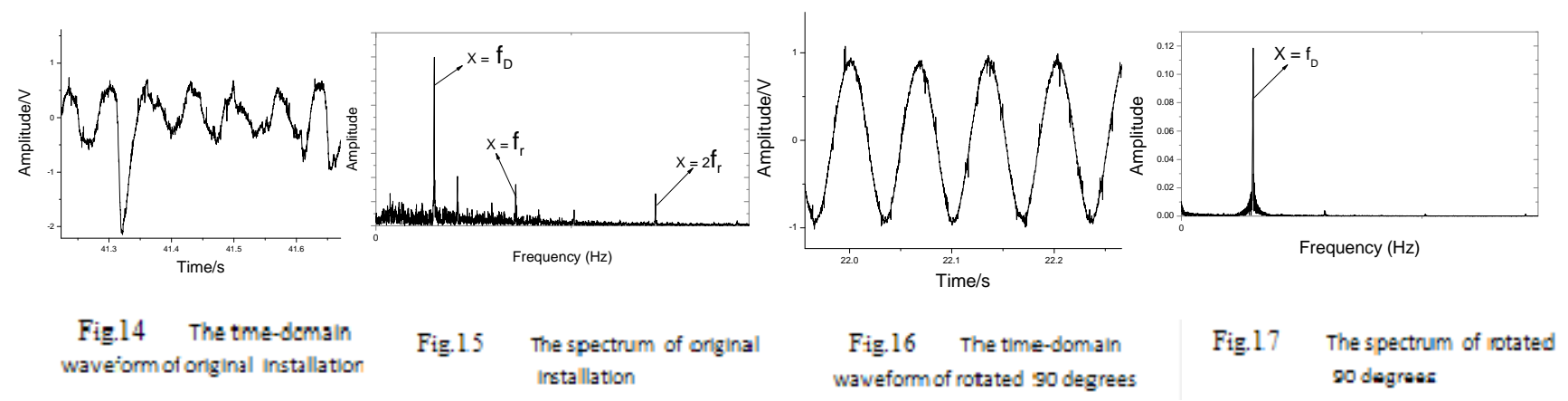

Comparatively analysis figures, it is obvious that time-domain waveform distortion was fully improved and resonant was effectively inhibited after rotate 90 degree. Signal to noise ratio (SNR) is also improved. The experimental results verify that the analysis and suppression method in this article are correct.

\section{Conclusion}

In this paper, with aircraft driven MEMS silicon micro-machined gyro as the research background, we study the dynamics of the silicon micro-machined gyro and the rotation aircraft rudder control mechanism. This paper analyzes the influence of the silicon micro-machined gyro output signal when the aircraft rudder surface deflection in the conventional installation. Aiming at this issue the paper puts forward installation method of rotation 90 degrees. The both of calculation and experimental results demonstrate the effectiveness of this method in the inhibition of the gyro output signal noise. By comparing the experimental results, it is shown that the proposed method can effectively eliminate the influence of the $f_{r}$ rudder partial force on the silicon micro-machined gyro, and improve the rate of qualified products. It has an important reference value of engineering application in the environment of the aircraft.

\section{Acknowledgment}

The project is sponsored by the National Natural Science Funds (60971024), The Beijing Natural Science Funds (4112020), Beijing Key Laboratory of Sensor Open Project (5026035209), and the Key Laboratory of Modern Measurement and Control Technology of the Ministry of Education. Thanks a lot for supporting.

\section{References}

[1] Zhang Fu-xue, Mao Xu, Zhang Wei, Si Micromachined Gyroscope Driven by the Rotating Carrier,China Engineering Science, vol. 8, No. 8, pp. 23 27, Aug. 2006.

[2] Meng Xiu-yun, The principle of missile guidance and control system, Beijing: Beijing Institute of Technology Press, 2003.

[3] Qian Xing-fang, Lin Rui-xiong, Zhao Ya-nan, Missile Flight Mechanics, Beijing: Beijing Institute of Technology Press, 2000.

[4] Zhang Fuxue, Zhang Wei, Silicon micromechanlcal gyroscope without driving structrue, GB 2449955A.

[5] Fuxue Zhang, Hongwei Wang, Wei Zhang, Yu Liu, Carrier-driven silicon micromachined gyroscope, ZL 200410028228.X.

[6] Liu Bao-zhu, Su Yan-hua, Zhang Hong-lin, MATLAB7.0 From Entry to the Master, Beijing: Posts and Telecom Press, 2010. 